\title{
IN-SILICO SCREENING AGAINST ANTIMALARIAL TARGET PLASMODIUM FALCIPARUM ENOYL-ACYL CARRIER PROTEIN REDUCTASE
}

\author{
BERWI FAZRI PAMUDI, AZIZAHWATI, ARRY YANUAR*
}

Department of Pharmaceutical Chemistry, Faculty of Pharmacy, Universitas Indonesia, Depok, Indonesia. Email: arry.yanuar@ui.ac.id Received: 21 April 2017, Revised and Accepted: 13 July 2017

\section{ABSTRACT}

Objective: Malaria is a parasitic infection that causes worldwide health problems. The absence of an effective vaccine and Plasmodium strains that are resistant to antimalarial drugs emphasize the importance of developing new chemotherapeutic agents. The use of computers for in-silico screening, or virtual screening, is currently being developed as a method for discovering antimalarial drugs. One of the enzymes that can support the development of the malaria parasite is the Plasmodium falciparum enoyl-acyl carrier protein reductase (PfENR). Inhibition of these enzymes leads to Type II lipid biosynthesis inhibition on the parasite.

Methods: This research investigates the use of virtual screening to find PfENR inhibitor candidates. A molecular docking method using GOLD software and the medicinal plants in Indonesia database will be used. This target has been optimized by the removal of residues and the addition of charge. Ligand is expected to be an inhibitor of PfENR.

Results: In-silico screening, or virtual screening, found that the top five compounds with the highest GOLD score at trial are kaempferol 3-rhamnosyl(1-3)-rhamnosyl-(1-6)-glucoside; cyanidin 3,5-di-(6-malonylglucoside); 8-hydroxyapigenin 8-(2", 4"'-disulfato glucuronide); epigallocatechin 3,5,-diO-gallat; quercetin 3,4'-dimethyl ether 7-alpha-L-arabinofuranosyl-(1-6)-glucoside. They had GOLD scores of 94.73, 95.90, 86.46, 85.39, and 84.40, respectively.

Conclusions: There are two candidate inhibitor compounds from tea (Camellia sinensis), which have potential for development as an antimalarial drug, which are kaempferol 3-rhamnosyl-(1-3)-rhamnosyl-(1-6)-glucoside and epigallocatechin 3,5,-di-0-gallate, with a GOLD score of 94.73 and 85.39 , respectively.

Keywords: Antimalarial, Camellia sinensis, In-silico screening, Plasmodium falciparum enoyl-acyl carrier protein reductase.

(C) 2017 The Authors. Published by Innovare Academic Sciences PvtLtd. Thisisan open access article under the CC BYlicense (http://creativecommons. org/licenses/by/4. 0/) DOI: http://dx.doi.org/10.22159/ajpcr.2017.v10s5.23114

\section{INTRODUCTION}

Malaria is one of the most serious parasitic infections in the world. There are more than 500 million cases of malaria per year, with about 3 million deaths. A total of $40 \%$ of the world's population is living in malaria-endemic areas and Indonesia, 35\% of the population live in areas at risk of malaria [1]. Malaria is the cause of hemolytic anemia due to infection of red blood cells by a protozoan of the genus Plasmodium transmitted to humans through the saliva of female Anopheles mosquitoes [2]. Four Plasmodium species cause malaria: Plasmodium falciparum, Plasmodium ovale, Plasmodium malariae, and Plasmodium vivax. Of these, four parasite species, P. falciparum is the source of the most severe and often fatal forms of the disease, tropical malaria, and cerebral malaria [3].

Antimalarial drugs, that are effective, safe, practical to use, and economically affordable are essential for reducing malaria deaths. Medications such as quinine, chloroquine, quinidine, mefloquine, and artemisinin derivatives are effective and work quickly; however, the problem, the world is facing today is parasite resistance to antimalarial drugs [1]. There are several possible reasons for malaria parasites to become resistant to antimalarial drugs like chloroquine. First, the parasite does not have an active site to bind with chloroquine, so this drug cannot be concentrated in the red blood cells; furthermore, resistant Plasmodium has other biochemical pathways which no heme involvement in the process to synthesize amino acids, and thus, avoid the influence of chloroquine. Chloroquine binds to heme in red blood cells forming the complex which is highly toxic resulting cell lysis and parasite autodigestion. Chloroquine is required in sufficient concentration in the red blood cells to act as antimalarial drug.
A second cause of resistance is the parasite's ability to spontaneously mutate under drug stress [4].

New, antimalarial drug development is essential to controlling malaria. To achieve this goal, identification of targets and compounds for inhibiting malaria treatment is needed [5]. In the last decade, considerable potential targets for antimalarials have been found. One general target includes pathways of Type II fatty acid biosynthesis, with a specific target, P. falciparum enoyl-acyl carrier protein reductase (PfENR), an enzyme that plays a significant role in Type II fatty acid biosynthesis of P. falciparum. PfENR catalyzes the last step of the elongation cycle of fatty acid biosynthesis and works by reducing the carbon double bond in the covalently bonded the acyl carrier protein [6,7]. The discovery of inhibitors of Type II fatty acid synthesis in the P. falciparum is expected to shorten the life cycle of the parasite. Terminations of biosynthetic pathways in the Plasmodium cause loss of the energy needed to perform metabolic processes and causing this organism to slowly die.

Advances in information technology today can offer alternatives to the search for new drugs. Therefore, screening methods used at an early stage in the search for drug candidates have been developed using computer technology. In this study, the screening process was conducted using virtual screening or in-silico (computer simulation). In-silico screening is a computing system or analog in-silico in biological screening. The purpose of in-silico screening is to look for value and rank or filter a set of data structures using one or more computational procedures. In-silico screening is used to help determine which compounds will be screened or help for the process of synthesis [8]. 


\section{METHODS}

Materials used in this study include three-dimensional (3D) structures of macromolecules, PfENR downloaded from the Protein Data Bank (http://www.rscb.org.pdb, PDB id: 1NHG), which binds to nicotinamide adenine dinucleotide and triclosan (TCL); the 3D structure of the ligand on the database of medicinal plants in Indonesia; and the positive and negative controls as PfENR inhibitor. PfENR, which is used for molecular docking targets, should be separated from the solvent and ligand or non-standard residues. Separation of molecules that are not required is done using the UCSF Chimera program. The results will be used for docking, which is then stored in the pdb format.

PfENR 3D structure optimization is performed using Vega ZZ software. Optimization includes the elimination of water molecules, the addition of a hydrogen atom, and repair charge by adding a Gasteiger partial charge, giving AutoDock force field, and the application of minimization. Macromolecule minimization is done in two stages, i.e., the 100 -steps steepest descent method, followed by the 1000 steps of conjugate gradients. Validation of virtual screening methods, or in-silico screening, was done by molecular docking. The structures of positive control (diazaborine and TCL) and negative control (ethionamide and isoniazid) are downloaded from http://pubchem.ncbi.nlm.nih.gov/and then perform the molecular docking optimization using the GOLD software.

Ligands used in this study were obtained from the database of medicinal plants in Indonesia [9], and totaled as many as 1450 in the form of 3D ligands with the Tripos/Sybyl mol2 format. Ligands were optimized using Vega ZZ software with the addition of hydrogen and energy minimization. Molecular docking of ligands from the Indonesian medicinal plants database with target molecules PfENR was performed with GOLD software. Molecular docking resulted in obtaining the top five ranking compounds according to the highest GOLD score. Ranked candidate inhibitor compounds were visualized using GOLD software and PyMOL.

\section{RESULTS AND DISCUSSION}

PfENRs have a specific sequence, beginning with the insertion of 43 amino acid residues on one strand that is not owned by other ENR species. This enzyme also has a complex area which is low in residues, between 325 and 367, which is surrounded by polar residues such as asparagine, lysine, glutamine, and serine [10]. The PfENR crystal structure shows that hydrophobic residues surround the active site: Val222, Tyr277, Tyr267, Phe368, Pro314, Gly313, Leu315, His214, Ala217, Asn218, Ala219, Lys220, Met231, and Lys285 [11].

To validate the virtual screening method to be used, the molecular docking with positive and negative control using genetic algorithms (GA) was run with several search option speeds, i.e. fast, medium, and slow (Table 1). Positive control showed better results compared to the negative control from the docking to PfENR. Each speed GA search option is performed five times per molecular docking. Coordinates used for docking space will also be used on the molecular docking in virtual screening. Coordinates obtained for the docking space coordinates (X, $\mathrm{Y}, \mathrm{Z})$ are $51.24,93.77$, and 34.53 , respectively.

Based on the results of in-silico screening, the top five compounds with the highest occurrence at trial were kaempferol3-rhamnosyl-(13)-rhamnosyl-(1-6)-glucoside; cyanidin 3; 5-di-(6-malonylglucoside); 8-hydroxyapigenin 8-(2", 4"-disulfato glucuronide); epigallocatechin 3,5,-di-O-gallat; and quercetin 3,4'- dimethyl ether 7-alpha-Larabinofuranosyl-(1-6)-glucoside with GOLD scores of 94.73, 95.90, 86.46, 85.39, and 84.40, respectively. GOLD score results showed the best position of PfENR inhibitor compounds compared with affinity energy. GOLD score assessment results are negative results from the sum of all energy, so that the greater the score, the better the bond [12]. Virtual screening results had many interactions with PfENR active site, that is, kaempferol 3-rhamnosyl-(1-3)-rhamnosyl-(1-6)-glycoside, and epigallocatechin 3,5,-di-0-gallate visualized and analyzed with using the GOLD program and PyMOL.
Kaempferol 3-rhamnosyl-(1-3)-rhamnosyl-(1-6)-glucoside showed a hydrogen bond with Leu315, Gly313, Leu265, and Gly106. Hydrogen bonding in the active site is due to hydrophobic interactions amplified by one or more hydrogen bonds. Hydrophobic interactions can be formed due to the active site PfENR surrounded by hydrophobic residues while the hydrogen bonds formed by amino acid residues electronegative groups such as- $\mathrm{OH}$ and $\mathrm{O} 29$ and 044. Kaempferol 3-rhamnosyl-(1-3)-rhamnosyl(1-6)-glucoside has a hydrogen binding between the hydroxyl group to the nitrogen and oxygen 029 at residue Leu315 at a distance of $2.339 \AA$ and $2.875 \AA$, and $2.917 \AA$ to Gly313 (Fig. 1). Residues Gly313 and Leu315 are located in a nicotinamide-binding pocket, thus binding on this residue leads to inhibition of the binding of the enzyme [10]. These barriers affect the substrate of PfENR that cannot occupy the active site of the enzyme.

Kaempferol 3-rhamnosyl-(1-3)-rhamnosyl-(1-6)-glucoside compound is a flavanol glycoside derived from the family of Theaceae plant species origin, i.e., Camellia sinensis, Camellia bohea, Camellia theifera, Thea sinensis, Thea assamica, Thea cochinchinensis, Thea cantoniensis, better known as tea. Flavonoids are known to have antibacterial activity, and some flavonoids have antimalarial activity [13]. Discovery and development of flavonoids as an antimalarial has been developed over the last few years $[13,14]$

Besides containing kaempferol 3-rhamnosyl-(1-3)-rhamnosyl-(1-6)glucoside, tea also contains a compound epigallocatechin 3,5,-di-0gallate. Sannella et al. [14] stated in his study that the catechins content in tea, especially epigallocatechin-3-gallate and epicatechingallate, have inhibitory activity on the growth of P. falciparum in vitro. Another study states that tea catechin has the same potential as the binding of TCL with PfENR. Epigallocatechingallate has strong binding with PfENR, to form a binary complex conformation [15].

Epigallocatechin 3,5,-di-0-gallate shows the hydrogen binding of electronegative groups such as $\mathrm{OH}$ on 016, 031, and 047 with Gly313, Tyr277, Asn218, with distances between 2.5 and $3.5 \AA$ A (Fig. 2). Similar to kaempferol 3-rhamnosyl-(1-3)-rhamnosyl-(1-6)-glucoside, which has the nicotinamide-binding pocket, epigallocatechin 3,5,-di-0-gallate also binds with Gly313 and Leu315 so it can inhibit the binding of the

Table 1: Average score of binding affinity (GOLD score) of positive control and negative control with PfENR from docking experiment with GOLD software

\begin{tabular}{lccc}
\hline Control & Fast & Medium & Slow \\
\hline Positive control & & & \\
$\quad$ Diazaborine & 45.38 & 48.82 & 50.24 \\
$\quad$ Triclosan & 43.09 & 43.49 & 43.59 \\
Negative control & & & \\
$\quad$ Ethionamide & 35.36 & 36.19 & 36.44 \\
$\quad$ Isoniazid & 31.86 & 32.63 & 32.55 \\
\hline PfENR: Plasmodium falciparum enoyl-acyl carrier protein reductase &
\end{tabular}

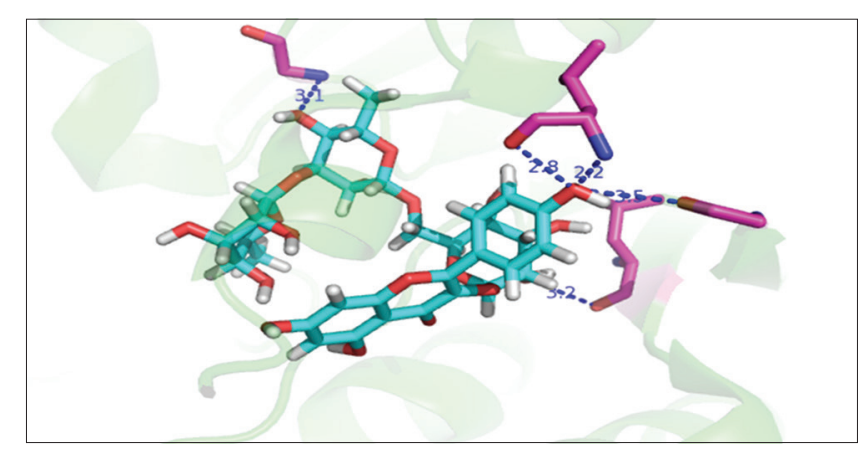

Fig. 1: Interaction of kaempferol 3-rhamnosyl-(1-3)- rhamnosyl(1-6)-glucoside (blue) with some amino acid residues (purple) on Plasmodium falciparum enoyl-acyl carrier protein reductase 


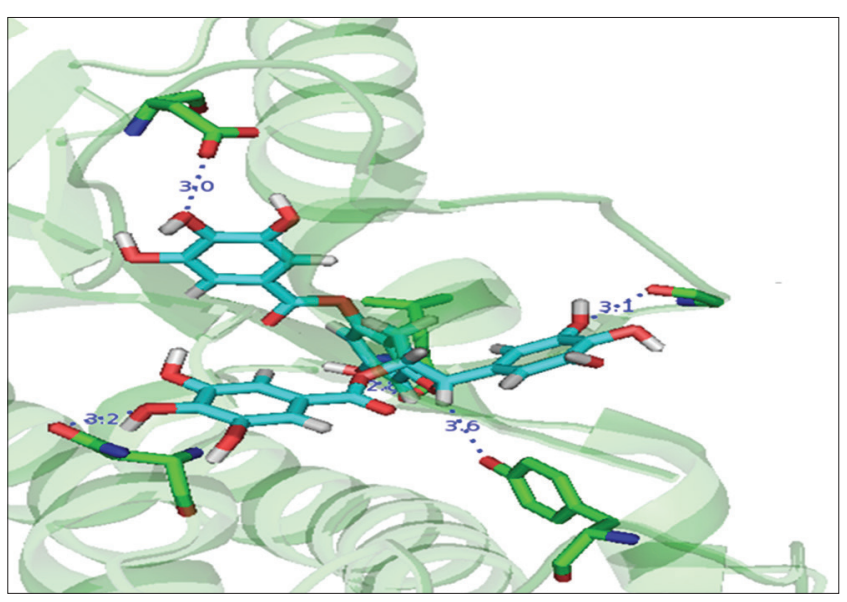

Fig. 2: Interaction of epigallocatechin 3,5,-di-0-gallate (blue) with some amino acid residues (green) in Plasmodium falciparum enoyl-acyl carrier protein reductase

substrate with the enzyme. This obstacle cannot make the enzyme work to catalyze reactions in the process of extending the synthesis of fatty acids on P. falciparum.

\section{CONCLUSION}

Based on the results of in-silico screening using the medicinal plants in Indonesia database against targets PfENR, there are two potential candidates of chemical compounds from tea ( $C$. sinensis) that have prospects as inhibitors: The chemical compounds kaempferol 3-rhamnosyl-(1-3)-rhamnosyl-(1-6)-glucoside and epigallocatechin 3,5,-di-0-gallate, with a GOLD score of 94.73 and 85.39, respectively.

\section{REFERENCES}

1. Harijanto PN, Nugroho A, Gunawan CA. Malaria Dari Molekuler ke Klinis. Jakarta: Penerbit Buku Kedokteran EGC ; 2009.
2. WHO. Guidelines for the Treatment of Malaria. Geneva: World Health Organization; 2010.

3. Katzung BG. Basic and Clinical Pharmacology. 10 $0^{\text {th }}$ ed. New York: McGraw-Hill Companies; 2006.

4. Pribadi W, Muljono R. Resistensi parasit malaria terhadap obat malaria. In: Gandahusada S, Ilahude H, Pribadi W, editors. Parasitologi Kedokteran. Jakarta: Gaya Baru; 2004. p. 197-8.

5. Mitamura T, Palacpac NM. Lipid metabolism in Plasmodium falciparum-infected erythrocytes: Possible new targets for malaria chemotherapy. Microbes Infect 2003;5(6):545-52.

6. Tasdemir D. Type II fatty acid biosynthesis, a new approach in antimalarial natural product discovery. Phytochem Rev 2006;5:99-108.

7. Kapoor M, Gopalakrishnapai J, Surolia N, Surolia A. Mutational analysis of the triclosan-binding region of enoyl-ACP (acylcarrier protein) reductase from Plasmodium falciparum. Biochem J 2004;381:735-41.

8. Leach AR, Shoichet BK, Peishoff CE. Prediction of protein-ligand interactions. Docking and scoring: Successes and gaps. J Med Chem 2006;49(20):5851-5.

9. Yanuar A, Mun'im A, Lagho AB, Syahdi RR, Rahmat M, Suhartanto H. Medicinal plants database and three dimensional structure of the chemical compounds from medicinal plants in Indonesia. Int J Comput Sci 2011;5(1):180-3.

10. Perozzo R, Kuo M, Sidhu AB, Valiyaveettil JT, Bittman R, Jacobs WR Jr, et al. Structural elucidation of the specificity of the antibacterial agent triclosan for malarial enoyl acyl carrier protein reductase. J Biol Chem 2002;277(15):13106-14

11. Moreno E. Structural detemination of triclosan derivatives as inhibitors of Plasmodium falciparum enoyl reductase (PfENR). Texas: Texas A and M University; 2005.

12. Nervall M, Hanspers P, Carlsson J, Boukharta L, Aqvist J. Predicting binding modes from free energy calculations. J Med Chem 2007;51:2657-67.

13. Cushnie T, Lamb A. Antimicrobial activity of flavonoids. Int $\mathrm{J}$ Antimicrob Agents 2005;26:343-56.

14. Sannella AR, Messori L, Casini A, Francesco Vincieri F, Bilia AR, Majori G, et al. Antimalarial properties of green tea. Biochem Biophys Res Commun 2007;353(1):177-81.

15. Banerjee T, Sharma SK, Surolia N, Surolia A. Epigallocatechin gallate is a slow-tight binding inhibitor of enoyl-ACP reductase from Plasmodium falciparum. Biochem Biophys Res Commun 2008;377(4):1238-42. 